Marquette University

e-Publications@Marquette

$5-1-2012$

\title{
A Density Functional Theory and Laser Flash Photolysis Investigation of Carbofuran Photodegradation in Aqueous Medium
}

\author{
A. Atifi \\ Marquette University \\ M. Talipov \\ H. Mountacer \\ Michael D. Ryan \\ Marquette University, michael.ryan@marquette.edu \\ M. Sarakha \\ Université Blaise Pascal
}

Follow this and additional works at: https://epublications.marquette.edu/chem_fac

Part of the Chemistry Commons

\section{Recommended Citation}

Atifi, A.; Talipov, M.; Mountacer, H.; Ryan, Michael D.; and Sarakha, M., "A Density Functional Theory and Laser Flash Photolysis Investigation of Carbofuran Photodegradation in Aqueous Medium" (2012).

Chemistry Faculty Research and Publications. 432.

https://epublications.marquette.edu/chem_fac/432 
Marquette University

e-Publications@Marquette

\section{Chemistry Faculty Research and Publications/College of Arts and Sciences}

This paper is NOT THE PUBLISHED VERSION.

Access the published version via the link in the citation below.

Journal of Photochemistry and Photobiology A: Chemistry, Vol. 235 (May 1, 2012): 1-6. DOI. This article is (C) Elsevier and permission has been granted for this version to appear in e-Publications@Marquette. Elsevier does not grant permission for this article to be further copied/distributed or hosted elsewhere without the express permission from Elsevier.

\section{A Density Functional Theory and Laser Flash Photolysis Investigation of Carbofuran Photodegradation In Aqueous Medium}

\section{A. Atifi}

Chemistry department, Marquette University, Milwaukee, Wisconsin

Laboratoire des Sciences de l'Environnement et du Developpement, Equipe de Chimie Ecologique, FST Settat, Morocco

M. Talipov

Chemistry department, Marquette University, Milwaukee, Wisconsin

H. Mountacer

Laboratoire des Sciences de l'Environnement et du Developpement, Equipe de Chimie Ecologique, FST Settat, Morocco

M.D. Ryan

Chemistry department, Marquette University, Milwaukee, Wisconsin

M.S arakha

Institut de Chimie de Clermont-Ferrand (ICCF) UMR6296, équipe de Photochimie, Université Blaise Pascal, 24, Avenue des, Landais, BP80026, 63171 Aubière, France 


\begin{abstract}
Density functional theory (DFT) approach was used to study the photodegradation of Carbofuran in aqueous medium. This computational method enables us to assign the electronic transitions and interpret the dissociative behavior upon irradiation based on a thermodynamical analysis of the bond dissociation energies (BDE) of Carbofuran. According to these calculations, phenoxy $\mathrm{C}_{-} \mathrm{O}$ bond appears weaker than the $\mathrm{C}_{-} \mathrm{N}$ bonds. Hence, it was predicted that the photodegradation of Carbofuran should occur with an initial homolytic dissociation of the C_O bond of the carbamate moiety. Laser Flash Photolysis (LFP) results clearly indicate the formation of the phenoxyl radical, which support the outcome of this theoretical approach.
\end{abstract}

\title{
Highlights
}

- A density functional theory for Carbofuran permitted to assign the electronic transitions and interpret the dissociative behavior upon irradiation. The photodegradation of Carbofuran mainly involves an initial homolytic dissociation of the $\mathrm{C}_{-} \mathrm{O}$ bond of the carbamate moiety. The Laser falsh photolysis experiments clearly indicate the formation of the phenoxyl radical derivative.

\section{Keywords}

DFT, Photodegradation, Carbofuran, BDE, Laser flash photolysis, Homolysis

\section{Introduction}

Carbofuran (2,3-dihydro-2,2-dimethyl benzofuran-7-yl methyl carbamate) is a broad spectrum insecticide, nematicide and miticide [1]. Residual fate of Carbofuran has been extensively investigated in water, soil and in different experimental conditions [2], [3], [4], [5]. In fact, several pathways were suggested for Carbofuran photodegradation, and the major observed photoproduct was phenol derivative [6]. Chiron et al. have published a report on Carbofuran photodegradation where two decomposition products were identified by LC-MS, 3-hydroxy-7-carbofuranphenol as a product of hydrolysis and 2-hydroxy-3-(2-methylprop-1-enyl)phenyl-N-methylcarbamate arising from a rearrangement product [7]. John and Howard have proposed a mechanism for the first steps of the Carbofuran photodegradation in water. According to these authors, the carbamate group undergoes a cleavage process via C_O heterolysis, leading to the formation of carbamic acid and 2,3-dihydro-2,2dimethylbenzofuran-7-ol [8]. However, the knowledge of the main photochemical reaction remains incomplete, and the mechanistic data are still needed. A mechanism of similar system is shown in Fig. 1 [9]. Upon irradiation, the generated excited singlet may undergo intersystem crossing (ISC) from $S_{1}$ to $T_{1}$, homolytic cleavage to form radical pair or heterolytic cleavage to form ion pair. The initially formed radical pair may be converted to the more stable (in polar solvents) ion pair by electron transfer (ET). Finally, both radical pairs and ion pairs may lead to formation of the starting substrate, namely carbofuran, within a cage recombination process: Radical Combination (RCom) or Ion Combination (ICom). 


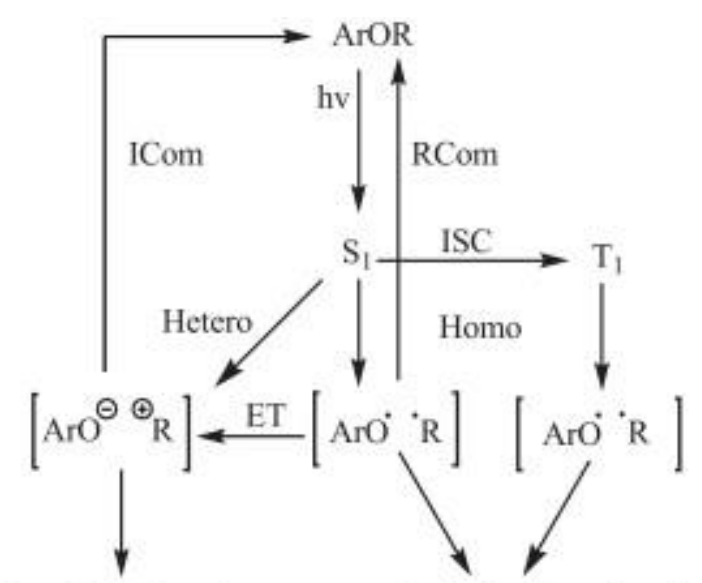

Ion-derived products Radical-derived products

Fig. 1. General mechanism of photodissociation.

On the other hand, quantum chemical computations have recently been considered as an effective tool for the analysis of pesticide molecules [10], [11], [12], [13], [14], [15]. Arul Dhas et al. have performed the density functional theory (DFT) computations to interpret electronic and vibrational spectra, and intramolecular charge transfer responsible for biological activity of Chlorothalonil [16]. Aaron and al. have applied a theoretical, gradient-corrected Hartree-Fock density functional theory (HF-DFT) approach to determine the bond dissociation energy (BDE) for the photodegradation processes of the 2,4-dichlorophenoxyacetic acid (2,4-D) herbicide in the gas phase and in aqueous medium [17].

In this context, the present work examines the mechanistic aspect of the first step of Carbofuran photochemical degradation in water, based on a theoretical approach using the DFT methods. In addition, our experimental data given by laser flash photolysis permit to corroborate this theoretical approach.

\section{Materials and methods}

\subsection{Experimental}

The compound Carbofuran (98) was purchased from Sigma-Aldrich. UV-vis spectrum was taken for aqueous sample of Carbofuran (0.1 mM), using the HP-8452A photo-diode array spectrometer in the region 190-700 nm.

Laser flash photolysis (LFP): Transient absorption experiments in the 20 ns to $500 \mu$ s time scale were carried out on a nanosecond laser flash photolysis spectrometer from Applied Photophysics (LKS.60). Excitation ( $\lambda=266 \mathrm{~nm})$ was from the fourth harmonic of a Quanta Ray GCR 130-01 Nd:YAG laser (pulse width $5 \mathrm{ns),} \mathrm{and} \mathrm{was} \mathrm{used} \mathrm{in} \mathrm{a}$ right-angle geometry with respect to the monitoring light beam. A $3 \mathrm{~cm}^{3}$ volume of an argon-saturated solution was used in a quartz cell, and was stirred after each flash irradiation. Individual cell samples were used for a maximum of 10 consecutive experiments. The laser energy was within the range 1-15 mJ. The obtained signal was an average of about 10 shots. The transient absorbance at preselected wavelength was monitored by a detection system consisting of a pulsed xenon lamp (150 W), monochromator, and a 1P28 photomultiplier. A spectrometer control unit was used for synchronizing the pulsed light source and programmable shutters with the laser output. This also housed the high-voltage power supply for the photomultiplier. The signal from the photomultiplier was digitized by a programmable digital oscilloscope (HP54522A). A 32 bits RISC-processor kinetic spectrometer workstation was used to analyze the digitized signal.

LC/MS studies were carried out with a Waters (Alliance 2695) high performance liquid chromatography system coupled to a Quattro LC triple quadrupole mass spectrometer (Micromass, Manchester, UK) equipped with a pneumatically assisted electrospray ionization source (ESI) and a Waters photodiode array detector. Data 
acquisition and processing were performed by MassLynx NT 3.5 system. Chromatography was run using a Nucleodur column100-5 C8 ec $(250 \times 4.6 \mathrm{~mm}, 5 \mu \mathrm{m})$ and a 60/40 (v/v) mixture of acetonitrile and water with $0.2 \%$ acetic acid as mobile phase at $1 \mathrm{~mL} \mathrm{~min}{ }^{-1}$. The electrospray source parameters were:capillary voltage $3.5 \mathrm{kV}$ (or $3 \mathrm{kV}$ in the negative mode), cone voltage $15 \mathrm{~V}$, source block temperature $120^{\circ} \mathrm{C}$, desolvatation gas temperature $400^{\circ} \mathrm{C}$. Argon was used for collisional activated dissociation (CAD) at a pressure of $1.5 \times 10^{-3}$ torr and $10-50 \mathrm{eV}$ collision energy.

The irradiations at $254 \mathrm{~nm}$ were obtained with PHILIPS TUV $6 \mathrm{~W}$ lamp delivering a parallel beam.

\subsection{Computational details}

The computation was performed using two density functional theory (DFT) based methodologies. The first methodology is based on the combination of the Beckes three-parameter adiabatic connection exchange functional (B3) with the Lee-Yang-Parr (LYP) correlation functional (B3LYP method) [18], [19]. The second DFT methodology, based on PBE1 exchange functional combined with the PBE correlation one10 (PBE0 method) is characterized by certain further improvements over the other DFT functional methods [20]. Indeed, generally the later methods are constructed with the main aim of improving the well-known deficiency in their long-range behavior. Both of the employed computational methodologies can be considered as hybrid HF-DFT (i.e. they include an admixture of HF exchange energy), in contrast to the pure DFT ones. The standard Pople-type 6$311++G(d, p)$ basis set was employed for orbital expansion, solving the Kohn-Sham (KS) SCF equations iteratively for each particular purpose of this study.

Electronic transitions for the molecule were calculated from excited state calculations using the time dependent-density functional theory (TD-DFT) method. This method is frequently found to be a powerful and accurate approach for describing low-lying excited states of conjugated molecules and has consequently been applied to solve many chemical and physical problems [21], [22], [23], [24].

The influence of the solvent on the energetics of bond dissociation processes for the species under study was accounted for in the framework of the self- consistent reaction field (SCRF) methodology, employing the Polarized Continuum Model (PCM) for water, the later medium being treated as a continuous dielectric with a relative dielectric constant (=78.39) [24], [25], [26]. In general, the PCM calculations were based on the gasphase structures optimized at the same theoretical level. Such method is used in many other works and is known to be consistent to study similar systems [27], [28].

Subsequently, after locating the stationary points on the potential energy surfaces (PESs) under study, their character was tested by computing the harmonic vibrational frequencies. The absence of imaginary frequencies (namely negative eigenvalues of the second-derivative matrix) was used as a criterion that a particular point on the PES corresponded to a minimum energy structure (instead of being a saddle point). Thermochemistry of the bond dissociation processes was analyzed in the following way, described below.

The energies for each of the involved species were corrected by the zero-point vibrational energies, computed on the basis of harmonic vibrational analysis. Subsequently, the rotational, vibrational and translational contributions were added to the obtained $E(0)$ values, in order to get the $E(T)$ ones (for $T=298.15 \mathrm{~K}$ ). All of these contributions were calculated on the basis of standard statistical mechanics expressions for an ideal gas constituted of harmonic oscillators within a canonical ensemble [29]. The corresponding enthalpies $H(T)$ and free enthalpies $G(T)$ were calculated from Eqs. (1), (2):

(1) $H(T)=E(T)+R T$

(2) $G(T)=H(T)-T S$ 
Finally, the bond dissociation reaction energies $E$ and enthalpies $H$ were calculated from the standard expressions for the corresponding quantities, given in Eqs. (3), (4), (5):

(3) $\Delta E^{0}=\sum_{p} E p-\sum_{r} E r$

(4) $\Delta H^{0}=\sum_{p} H p-\sum_{r} H r$

(5) $\Delta G^{0}=\sum_{\mathrm{p}} G p-\sum_{\mathrm{r}} G r$

where the indices $p$ and $r$ represent, respectively, the products and the reactants of the processes under study.

All quantum chemical calculations for the purpose of the present study were carried out with the Gaussian 09 series.

\section{Results and discussion}

\subsection{Geometry optimization}

Optimized structure of Carbofuran, using the atomic numbering, is shown in Fig. 2. The relevant geometry parameters have been calculated for Carbofuran and the relevant species arising from the dissociation pathways under study, which correspond to minimas on the B3LYP and PBE0/6-311++G(d,p) PESs in water environment. Cartesian coordinates of all optimized structures are included in the supporting material.

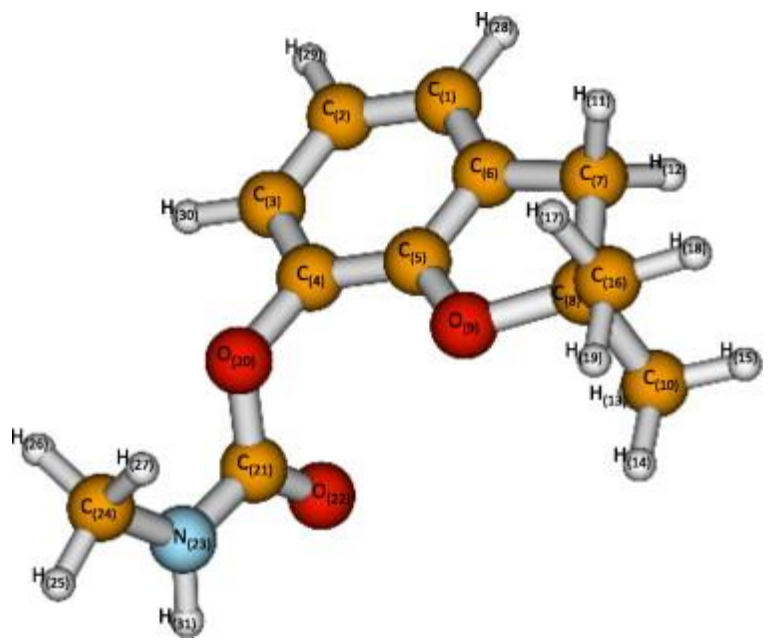

Fig. 2. Optimized structure and atomic numbering of Carbofuran at DFT level calculation.

\subsection{TD-DFT and UV-vis spectra}

Electronic transitions are usually classified according to the orbitals engaged or to specific parts of the molecule involved. Common types of electronic transitions in organic compounds are $\pi-\pi^{*}$ and $n-\pi^{*}$. In order to understand those transitions in Carbofuran molecule, TD-DFT calculation on electronic absorption spectrum was performed. The UV absorption bands in Carbofuran are observed at 203, 218 and $274 \mathrm{~nm}$. The TD-DFT calculations show three electronic transitions with significant oscillators strength, which are in good agreement with experimental values (see Table 1). The similarity of the spectra permitted to corroborate the used of the adopted method/basis set for this study. In attempt to characterize the electronic character of those transitions, 
we analyzed the involved molecular orbitals. It appears that all observed bands are $\pi-\pi^{*}$ transitions.
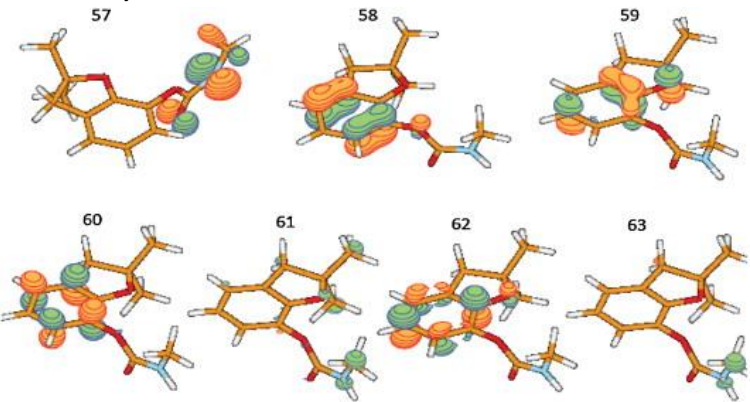

Molecular orbitals involved in electronic transitions of carbofuran

Table 1. TD-DFT calculations of Carbofuran.

\begin{tabular}{|l|l|l|l|l|l|l|}
\hline 6-311++G(d,p) & & B3LYP & & PBE0 & & Exp \\
\hline Excited state & MOs $^{\text {a }}$ & $\boldsymbol{\lambda} \mathbf{( n m )}$ & $\boldsymbol{f}^{\text {b }}$ & $\boldsymbol{\lambda} \mathbf{( n m )}$ & $\boldsymbol{f}^{\text {b }}$ & $\boldsymbol{\lambda}(\mathbf{n m})$ \\
\hline E1 & $58_{(\pi)}-62_{\left(\pi^{*}\right)}$ & 257 & 0.0811 & 251 & 0.0825 & 274 \\
\hline & $59_{(\pi)}-60_{\left(\pi^{*}\right)}$ & & & & & \\
\hline E3 & $59_{(\pi)}-61_{\left(\pi^{*}\right)}$ & 225 & 0.0462 & 220 & 0.0439 & 218 \\
\hline & $59_{(\pi)}-61_{\left(\pi^{*}\right)}$ & & & & & \\
\hline & $57_{(\pi)}-60_{\left(\pi^{*}\right)}$ & & & & & \\
\hline & $58_{(\pi)}-60_{\left(\pi^{*}\right)}$ & & & & & \\
\hline & $58_{(\pi)}-61_{\left(\pi^{*}\right)}$ & 196 & 0.092 & 197 & 0.3509 & 203 \\
\hline & $58_{(\pi)}-62_{\left(\pi^{*}\right)}$ & & & & & \\
\hline & $59_{(\pi)}-62_{\left(\pi^{*}\right)}$ & & & & & \\
\hline
\end{tabular}

${ }^{a}$ Molecular orbitals.

${ }^{\mathrm{b}}$ Oscillator strength.

\subsection{Thermodynamical analysis}

Based on a HF-DFT approach we have calculated by the B3LYP and the PBEO methods the bond dissociation reaction energies (BDE) and enthalpies of the dissociation asymptotes corresponding to the homolytic and heterolytic photocleavage processes for Carbofuran in water. These thermodynamical analysis deals with ground state of short-lived intermediates. The computed thermochemical parameters characterizing the bond dissociation processes of Carbofuran are presented in Table 2.

Table 2. Computed thermochemical parameters of Carbofuran in aqueous medium. Parameters are given in $\mathrm{kJ} / \mathrm{mol}$.

\begin{tabular}{|l|l|l|l|l|l|l|l|l|l|}
\hline 6-311++G(d,p) & B3LYP & & & & & PBE0 & & & \\
\hline Cleavage & Bond & $\boldsymbol{\Delta E}$ & $\boldsymbol{\Delta T E}$ & $\boldsymbol{\Delta H}$ & $\boldsymbol{\Delta} \boldsymbol{G}$ & $\boldsymbol{\Delta} \boldsymbol{E}$ & $\boldsymbol{\Delta T E}$ & $\boldsymbol{\Delta} \boldsymbol{H}$ & $\boldsymbol{\Delta} \boldsymbol{G}$ \\
\hline Homolytic & $\mathrm{O}_{(20)}-\mathrm{C}_{(21)}$ & 226.3 & 225.6 & 228 & 173.2 & 255.1 & 254.4 & 256.9 & 202.3 \\
\hline & $\mathrm{C}_{(21)}-\mathrm{N}_{(23)}$ & 385.6 & 386.3 & 388.8 & 335.8 & 410.4 & 411.2 & 413.7 & 360.5 \\
\hline & $\mathrm{N}_{(23)}-\mathrm{C}_{(24)}$ & 359.4 & 362.8 & 365.3 & 314 & 378.8 & 382.4 & 384.9 & 332.9 \\
\hline Heterolytic & $\mathrm{O}_{(20)-\mathrm{C}_{(21)}}$ & 269.6 & 268.2 & 270.7 & 222 & 295.4 & 294.1 & 296.6 & 247.1 \\
\hline & $\mathrm{C}_{(21)}-\mathrm{N}_{(23)}$ & 560.1 & 561.1 & 563.6 & 512.1 & 597.7 & 598.5 & 601 & 550.7 \\
\hline & $\mathrm{N}_{(23)}-\mathrm{C}_{(24)}$ & 498.5 & 500.1 & 502.5 & 458.4 & 531.1 & 532.9 & 535.4 & 488 \\
\hline
\end{tabular}


These theoretical, thermochemical parameters imply the following interpretation for the occurrence of the photodegradation mechanism of Carbofuran in an aqueous medium.

In fact, the homolytic bond dissociation energies are lower than heterolytic ones. Therefore, homolytic photocleavage of the different bonds should be promoted than heterolytic processes. Moreover, the homolytic BDE of the $C_{-} \mathrm{O}$ bond is lower than the $\mathrm{C}_{-} \mathrm{N}$ BDEs ones. This important thermodynamical argument would favor the $\mathrm{C}_{-} \mathrm{O}$ dissociation asymptote over the $\mathrm{C} \_\mathrm{N}$ ones. These preferential processes are also in agreement with other works [30], [31], [32]. As reported for photolysis of carbamates in water, the process should occur with an initial homolytic cleavage of the phenoxy bond.

\subsection{Laser flash photolysis}

In order to detect the transient species generated under light excitation we performed laser flash experiment using the fourth harmonic of a Nd:YAG laser ( $266 \mathrm{~nm}$ ). The laser excitation of oxygen free aqueous solution of carbofuran at the concentration of $5.0 \times 10^{-3} \mathrm{~mol} \mathrm{~L}^{-1}$ leads the detection of transient species that absorbs within the wavelength regions of $290 \mathrm{~nm}$ and $450 \mathrm{~nm}$ (Fig. 3). The decay kinetics was the same within the whole wavelength region suggesting the presence of a single intermediate species [33], [34]. It exhibits well-defined absorptions at $380 \mathrm{~nm}$ and $420 \mathrm{~nm}$ and was assigned unequivocally to the phenoxyl radical derivative based and the similarity with the spectra reported for this type of phenoxyl intermediates. The decay kinetics of the observed species was monitored at $420 \mathrm{~nm}$ and shows that the generated phenoxyl radical presents a half-life of about $1.5 \mu$ s in oxygen free solutions (Fig. 3, inset). Such half-life appears to be too short for a phenoxyl radical therefore suggesting that its decay involves reactions with other radicals generated in the system. Moreover, it is important to note that the absorbance of this transient species increased linearly with the laser energy within the range 2-10 $\mathrm{mJ}$ indicating its formation through a monophotonic process (Fig. 4, inset). A non-negligible oxygen effect was clearly observed on the decay of phenoxyl radical. The pseudo first order rate constant under oxygen saturated aqueous solution was evaluated to $4.0 \times 10^{6} \mathrm{~s}^{-1}$ (Fig. 4). It is worth noting that under our experimental conditions, no significant absorption was observed within the region $600-800 \mathrm{~nm}$ suggesting that no solvated electron is formed through a photoejection process.

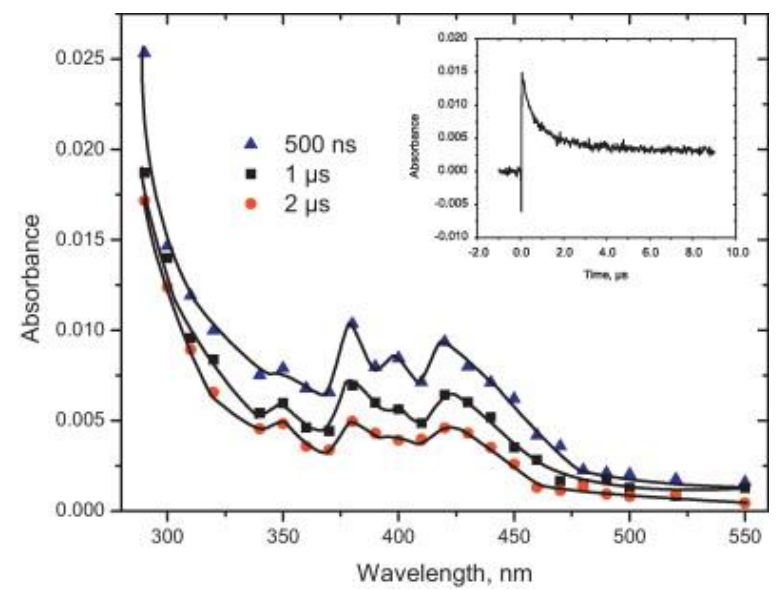

Fig. 3. Transient spectra showing phenoxyl radicals after laser flash photolysis $(266 \mathrm{~nm})$ of Carbofuran in aqueous solutions, monitored $500 \mathrm{~ns}, 1 \mathrm{~s}$ and $2 \mathrm{~s}$ after laser excitation. Inset: phenoxyl transient decay trace recorded after laser flash photolysis $(266 \mathrm{~nm})$, monitored at $400 \mathrm{~nm}$. 


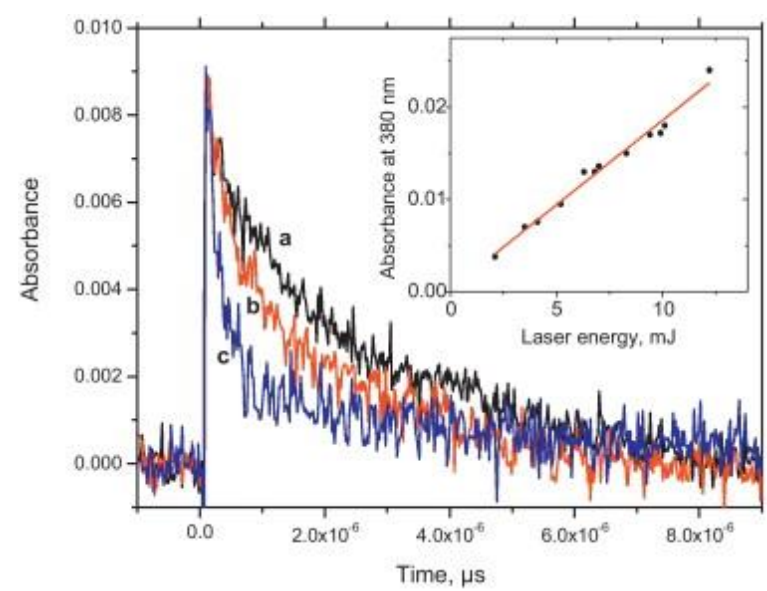

Fig. 4. Oxygen effect on the phenoxyl radical decay at $\lambda=400 \mathrm{~nm}$ (a) oxygen free solution; (b) aerated solution; and (c) oxygen saturated solution. The insert shows the dependence of the absorbance at $\lambda=380 \mathrm{~nm}$ with laser energy.

\subsection{Products analysis}

The characterization of some byproducts of a solution $3.0 \times 10^{-4} \mathrm{~mol} \mathrm{~L}^{-1}$ of carbofuran irradiated at $254 \mathrm{~nm}$ and $\mathrm{pH}=5.4$ was achieved by HPLC/ESI/MS technique. As described in the experimental section, the mass spectrometry experiments were performed in both positive and negative ion modes. The positive mode was found to be more suitable method for the parent compound and also for the of photogenerated products. An irradiated solution presenting $20 \%$ conversion showed the presence of remaining carbofuran with $[\mathrm{M}+1]=222$ and several byproducts. At least one isomer presenting the same molecular ion mass that corresponds more likely to a structure with a different position of the group function $\mathrm{CO}_{-} \mathrm{NH}_{-} \mathrm{CH}_{3}$. The presence of the phenolic derivative was also detected at shorter retention time with a molecular ion of $[\mathrm{M}+1]=165$. Moreover, trace concentration of a hydroquinone form was also detected with $[\mathrm{M}+1]=181$. The irradiation within the $\mathrm{pH}$ range 3-7 did not show any changes concerning the nature of the generated byproducts.

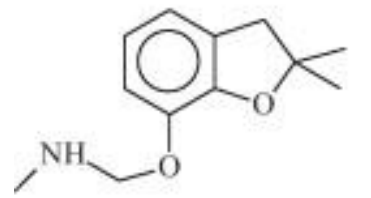

$[\mathrm{M}+1]=222$

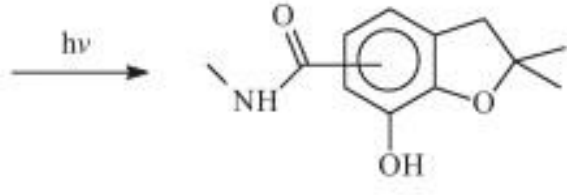

$[\mathrm{M}+1]=222$

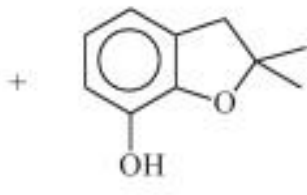

$[\mathrm{M}+1]=165$

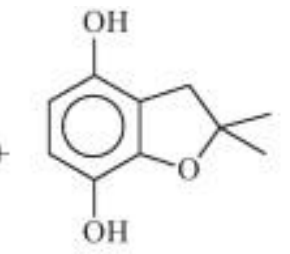

$[\mathrm{M}+1]=181$

\subsection{Mechanism}

As already demonstrated, the BDE calculations imply an initial homolytic photocleavage of the Phenoxy bond of Carbofuran molecule. This prediction is in complete agreement with laser flash photolysis findings. These clearly show that under direct irradiation of Carbofuran in aqueous solution the degradation involves the formation of the phenoxyl radical.

It should be noted that, although photolysis involves the chemistry of excited state which requires a high level of theoretical calculations, our simple approach taking into account only the chemistry of ground state has allowed us to predict the photochemical behavior of Carbofuran in water.

Considering the outcome of this investigation, we propose a mechanism Fig. 5 for the first step of Carbofuran photodegradation in water. This mechanism consists on an initial homolytic cleavage of the carbamate moiety, 
after light illumination, Carbofuran singlet excited state permits the formation of a radical pair in the solvent cage, which then undergoes intermolecular coupling or side reactions.

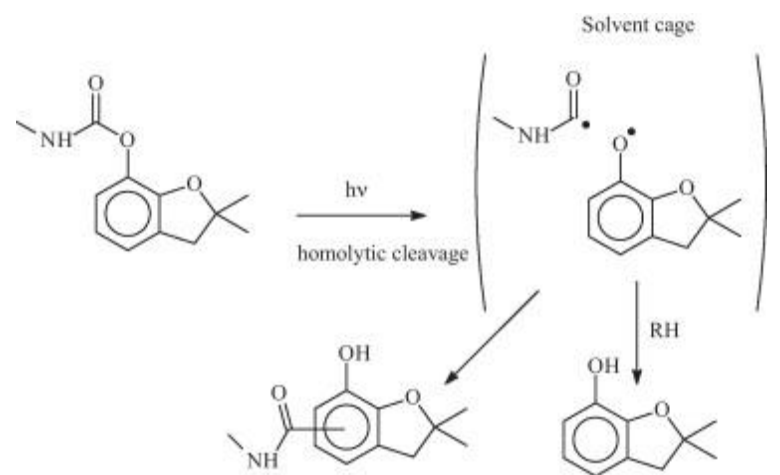

Fig. 5. Mechanism for the first step of Carbofuran photodegradation in water.

The photo-Fries rearrangement mechanism is well documented to occur in aryl esters, amides and carbamates [35]. It is also reported in other studies on photodegradation of Carbofuran and similar molecule [36], finally, to form carbofuran phenol and carbamic acid, we believe that these radicals must undergo side reactions.

The action of molecular oxygen on the solvent cage will lead first to a cage escape process (Fig. 6, Eq. (1)) that is in favor of an efficient production of the phenoxyl radical and second to the formation of the quinone derivative by its reaction on the para position of the aromatic moiety (Fig. 6, Eqs. (2), (3)). The mechanism may be proposed as shown in Fig. 6.

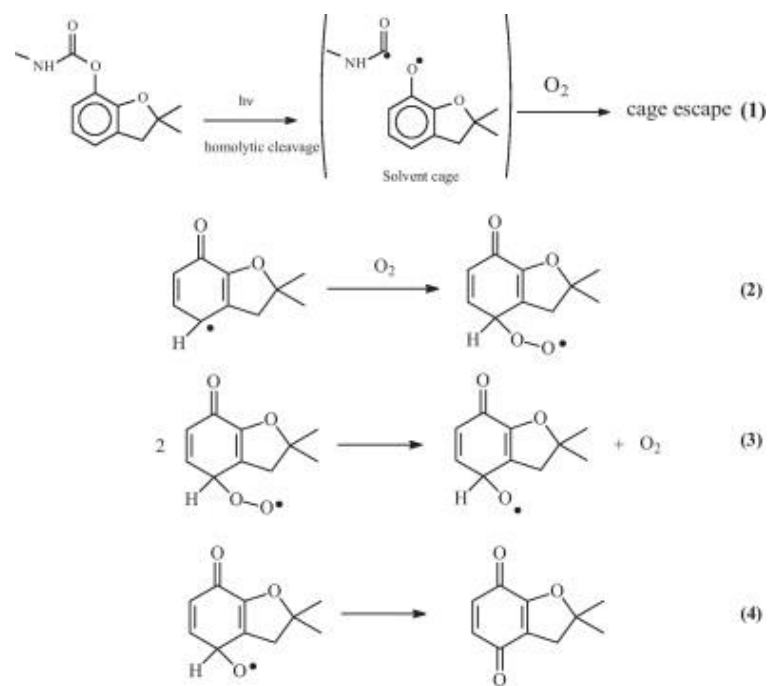

Fig. 6. Proposed mechanism for the role of oxygen in the formation of quinone derivative.

\section{Conclusion}

This investigation into the photochemistry of pesticides suggests that the photodecomposition pathways of Carbofuran, in aqueous medium, could be predicted by the density functional theory approach. The thermodynamical analysis, based on Bond Dissociation Energies comparison, implies an initial homolytic photodissociation of Phenoxy bond of Carbofuran molecule. Further, it is interesting to emphasize that these theoretical thermodynamic calculations are well corroborated by our experimental, Laser flash 
photolysis, results which reveals the formation of phenoxyl radical upon direct irradiation of Carbofuran in water.

\section{Acknowledgments}

The authors would like to thank the North Atlantic treaty Organization (NATO)for the financial support through the Collaborative LInkage Grant (CBP.MD.982508). The authors are grateful to FULBRIGHT Organization for their support and to Marquette University for using the Pere Cluster. The authors thank Dr. Q. Timerghazin for his collaboration.

\section{References}

[1] J. Tannock, C.L. Wessels. Pestic. Sci., 12 (1981), pp. 228-234

[2] F.J. Benitez, J. Beltran-Heredia, T. Gonzalez, F. Real. Ind. Eng. Chem. Res., 34 (1995), pp. 4099-4105

[3] M.J. Mansour, E.A. Feicht, A. Behechti, I. Scheunert. Chemosphere, 35 (1997), pp. 39-50

[4] Li-An Lu, Ying-Shih Ma, Mathava Kumar, Jih-Gaw Lin. Chem. Eng. J., 166 (2011), pp. 150-156

[5] J. Wei, G. Furrer, S. Kaufmann, R. Schulin, Environ. Sci. Technol., 35 (2001), pp. 2226-2232

[6] V. Samanidou, K. Fytianos, G. Pfister, M. Bahadir. Sci. Total Environ., 76 (1988), pp. 85-92

[7] S. Chiron, J.A. Torres, A. Fernandez-Alba, M.F. Alpendurada, D. Barcelo. D. Int. J. Anal. Chem., 65 (1996), pp. 37-52

[8] J. Bachman, H.H. Patterson. Environ. Sci. Technol., 33 (1999), pp. 874-881

[9] Alexandra L. Pincock, James A. Pincock, Roumiana Stefanova. J. Am. Chem. Soc., 124 (33) (2002), pp. 97689778

[10] E.J. Delgado. Chem. Phys. Lett., 471 (2009), pp. 133-135

[11] L. Zhang, G.-F. Hao, Y. Tan, Z. Xi, M.-Z. Huang, G.-F. Yang. Bioorg. Med. Chem., 17 (2009), pp. 4935-4942

[12] W. Yang, Z. Qian, B. Lu, J. Zhang, S. Bi. Geochim. Cosmochim. Acta (2010), pp. 1220-1229

[13] V. Arjunan, I. Saravanan, P. Ravindran, S. Mohan. Spectrochim. Acta, A74 (2009), pp. 642-649

[14] V.L. Furer, I.I. Vandyukova, A.E. Vandyukov, S. Fuchs, A.E. Vandyukov. Spectrochim. Acta, A74 (2009), pp. 642-649

[15] V. Mukherjee, N.P. Singh, R.A. Yadav. Spectrochim. Acta, A73 (2009), pp. 249-256

[16] D. Arul Dhas, I. Hubert Joe, S.D.D. Roy, T.H. Freeda. Spectrochim. Acta A, 77 (2010), pp. 36-44

[17] A. Jean-Jacques, I.G. Sandrine, LjupcoPejov, E. Sneana, Zdravkovski Zoran. Croat. Chem. Acta, 83 (2) (2010), pp. 171-177

[18] A.D. Becke. Phys. Rev., A38 (1988), pp. 3098-3100

[19] C. Lee, W. Yang, R.G. Parr. Phys. Rev. B, 37 (1988), pp. 785-789

[20] J.P. Perdew, K. Burke, M. Ernzerhof. Phys. Rev. Lett., 77 (1996), pp. 3865-3868

[21] R. Bauernschmitt, R. Ahlrichs. Chem. Phys. Lett., 256 (1996), p. 454

[22] M.E. Casda, C. Jamorski, K.C. Casida, D.R. Salahub. J. Chem. Phys., 188 (1998), p. 4439

[23] R.E. Stratmann, G.E. Scuseria, M.J. Frisch. J. Chem. Phys., 109 (1998), p. 8218

[24] M. Cossi, V. Barone. J. Chem. Phys., 115 (2001), p. 4708

[25] S. Miertus, E. Scrocco, Tomasi. J. Chem. Phys., 55 (1981), p. 117

[26] V. Barone, M. Cossi. J. Chem. Phys., 107 (1997), p. 3210

[27] C. Lacaze-Dufaure, F. Najjar, C. Andr-Barres. J. Phys. Chem. B, 114 (2010), pp. 9848-9853

[28] M. Leopoldini, F. Rondinelli, N. Russi, M. Toscana. J. Agric. Food Chem., 58 (2010), pp. 8862-8871

[29] D.A. McQuarrie. Statistical Mechanics. Harper and Row, New York, USA (1976)

[30] R. Givens. Org. Photochem. Photobiol., 69 (2004)

[31] Su Gi. J. Agric. Food Chem. (1972), p. 642

[32] V. Ramamurthy, S. Kirk, Schanze. Org. Mol. Photochem. (2003), p. 254

[33] J. Andraos, G.C. Barclay, D.R. Medeiros, M.V. Baldovi, J.C. Scaiano, R. Sinta. Chem. Mater., 10 (1998), pp. 1694-1699 
[34] Ana. Sanjuna, Guillermo, Aguirreb, Mercedes, Guillermo, Hermenegildo, J.C. Garca, Scaiano. Appl. Catal. B: Environ., 25 (2000), pp. 257-265

[35] A. Miranda, H. Garcia. Rearrangements in the Chemistry of Acids Derivatives. Wiley, New York (1991) pp. 1271-1394

[36] Bhattacharya Amitava, P. Raha, Asit K. Das, N. Adityachaudhury. Chemosphere, 29 (2) (1994), pp. 155-162 